\title{
Time Evolution of Infinite Classical Systems with Singular, Long Range, Two Body Interactions
}

\author{
Errico Presutti $\star$ \\ Istituto Matematico, Università dell'Aquila, I-67100 L'Aquila, Italy \\ Mario Pulvirenti and Brunello Tirozzi $\star \star$ \\ Istituto Matematico Università di Camerino, I-62032 Camerino, Italy
}

\begin{abstract}
Existence of dynamics for infinitely many hard-spheres in $v$ dimensions is proven in a set of full equilibrium measure.

Singular unbounded perturbations are considered with pair potentials diverging as $(x-a)^{-\lambda}, \lambda>2$ and $a$ is the hard-core diameter. Long range forces are allowed with potentials decreasing at infinity as $x^{-\lambda^{\prime}}, \lambda^{\prime}>v$. The result corrects and generalizes a proof given in a previous paper by the same authors.
\end{abstract}

\section{Introduction}

This is a revised and corrected version of a previous paper [1] by the same authors. In that work the existence of dynamics for infinitely many one dimensional hardrods was considered. Since then many results have been obtained, dynamics has been proven to exist in more general cases [2-4] and therefore one of the aims of that paper, to provide clues to the many dimension extension is no more actual.

However the techniques so far used [1-5] either required a Lipschitz assumption on the pair potential $[3,4]$ or a probabilistic (statistical) proof that dynamics is essentially finite, in the sense that the particles are grouped into finite, mutually non interacting clusters $[2,5]$.

The purpose of this paper is to exploit a method used in [1] to relax the Lipschitz condition on the pair potential and to prove the existence of dynamics without any finite cluster consideration: therefore no restriction is required on the range of values of temperature and chemical potential. In this paper we treat pair potentials $\Phi(r)$ which suitably diverge at the hard-core distance, we need the [presumably technical] condition that $\Phi(r)$ behaves as $(r-a)^{-\lambda}, \lambda>2$ for $r \rightarrow a$. Long range potentials are allowed, see D 2.2.

Since our approach applies to the many dimensional case as well, this is what we treat here: no main difference in procedure exists with respect to the onedimensional case.

* Research partially supported by a CNR fellowship Posit. 204530.

$\star \star$ Research partially supported by a CNR fellowship.

Present address: USSR Academy of Sciences. Leniuskij Prospect 14, Moskva B 71, USSR. 
The line of the proof is the same as in [3]. The only difficulty lies on the fact that since the derivative of the force diverges when the hard cores come close to each other, the Lipschitz constant cannot be defined directly. The idea is that it is the same interaction that prevents this to happen and that as a matter of fact during the motion the force is bounded and a Lipschitz constant can still be introduced. This would seem to mean that an a priori knowledge of the existence of dynamics is required, but as in [1-5] we can first consider partial flows in which only a finite number of particles move. The infinite case is then finally obtained by a limiting procedure. An estimate on the closeness of the particles is then the main requirement needed. It is essentially derived by energy considerations: particles have small probability to be initially too close because the energy becomes large and the Gibbs factor decreases. For the partial flows the measure is invariant and the same considerations can be extended to later times.

This qualitative argument can be made rigorous by the Ambrose Kakutani representation of flows technique [6], as first used by Sinai [5], in this context, in orded to bound the velocities of the particles. The setback in this procedure is that we cannot characterize explicitely the set of "good" configurations nor we can exhibit any "bad" configuration at all. It is only in the one-dimensional case with bounded short range forces that, with a different procedure [7], it has been possible to determine explicitely the configurations for which time evolution exists.

In Section 2 we introduce notations and definitions and we state the main result. In Section 3 we introduce the necessary statistical estimates. In Section 4 we give a proof of the existence theorem. In an appendix we give a (sketchy) proof of the statistical estimates of Section 3 and of some minor lemmas needed in Section 4.

\section{Definitions and Results}

In this section we give the main definitions and notations used throughout this paper and state the main theorem.

\section{D2.1 Phase Space. $\mathfrak{X}$ is the phase space of infinitely many hard-cores}

$$
x=\left(\hat{q}_{x}, \hat{p}_{x}\right) \in \mathfrak{X} \quad \hat{q}_{x}=\left(\ldots q_{i} \ldots\right) \quad \hat{p}_{x}=\left(\ldots p_{i} \ldots\right) \quad q_{i}, p_{i} \in \mathbb{R}^{v}
$$

where $q_{i}$ is the position of the " $i$ " particle and $p_{i}$ its momentum. Therefore $\left|q_{i}-q_{j}\right| \geqq a$, where $a$ is the hard-core length.

A topology is introduced in $\mathfrak{X}$ in the usual way $[8,3]$.

If $\Delta$ is a (Lebesgue measurable) region in $\mathbb{R}^{v}, \mathfrak{X}(\Delta)$ is the subset of $\mathfrak{X}$ with no particle outside $\Delta$.

D2.2 Interactions. $\Phi$ is the pair interaction between particles. $\Phi$ depends on the distance between them and we require it satisfies the following conditions:

$\Phi(x)$ is a $C^{2}$ function in $(a, \infty)$,

for $x \leqq a \Phi(x)=\infty$,

for $a \leqq x \leqq r_{1} \Phi(x) \geqq(x-a)^{-\lambda_{1}}\left|\Phi^{\prime}(x)\right| \leqq(x-a)^{-1-\lambda_{2}},\left|\Phi^{\prime \prime}(x)\right| \leqq(x-a)^{-2-\lambda_{2}}$, for $r_{2} \leqq x|\Phi(x)| \leqq x^{-\lambda^{\prime}}\left|\Phi^{\prime}(x)\right| \leqq x^{-1-\overline{\lambda^{\prime}}}\left|\Phi^{\prime \prime}(x)\right| \leqq x^{-2-\lambda^{\prime}}$.

We consider the case $\lambda_{2} \neq \lambda_{1}$ in order to allow potentials not necessarily increasing as $(x-a)^{-\lambda}$. In the proof of the existence of dynamics we will need the following 
condition on $\lambda_{1}$ and $\lambda_{2}$

$$
u=\left(2 \lambda_{1}\right)^{-1}\left(2+\lambda_{2}\right)<1 \text {. }
$$

This gives in the case $\lambda_{1}=\lambda_{2}=\lambda, \lambda>2$.

We will also need that $\lambda^{\prime}>v$ where $v$ are the dimension of the space in which the particles move.

D2.3 Equilibrium Measure. The following equilibrium equations define the equilibrium measure $\mu,[9]$. For $\Delta \subset \mathbb{R}_{v}$ bounded and Lebesgue measurable

$$
\begin{aligned}
\int_{\mathfrak{X}} \mu(d x) f(x)= & \int_{\mathfrak{X}\left(\Delta^{c}\right)} \mu(d x) \sum_{n=0}^{\infty}(n !)^{-1} \int_{\Delta^{n}} d(q)_{n} \int_{\mathbb{R}^{v n}} d(p)_{n} \exp \left\{-\beta H\left[(q)_{n}(p)_{n} \mid x\right]\right\} \\
& \cdot f\left[(q)_{n}(p)_{n} x\right]
\end{aligned}
$$

where $f$ is a $L^{1}(\mathfrak{X}, \mu)$ function and

$$
d(q)_{n}=d q_{1} \ldots d q_{n}, \quad d(p)_{n}=d p_{1} \ldots d p_{n} \exp (\beta \bar{\mu} n)
$$

[ $\bar{\mu}$ is the chemical potential]. $\beta=(k T)^{-1}, T$ is the temperature

$$
\begin{aligned}
H\left[(q)_{n}(p)_{n} \mid x\right] & =T\left[(p)_{n}\right]+U\left[(q)_{n} \mid x\right] \\
T\left[(p)_{n}\right] & =\sum_{i}^{n}(2 m)^{-1} p_{i}^{2} \\
U\left[(q)_{n} \mid x\right] & \left.\left.=U[q]_{n}\right]+I[q)_{n} \mid x\right] \\
U\left[(q)_{n}\right] & =\sum_{i \neq j} \phi\left(\left|q_{i}-q_{j}\right|\right) \\
I\left[(q)_{n} \mid x\right] & =\sum_{q_{j} \in \hat{q}_{x}} \sum_{1}^{n} \Phi\left(\left|q_{j}-q_{i}\right|\right) .
\end{aligned}
$$

D2.4 Equations of Motion. The equations of motion formally read as

$$
\begin{aligned}
& m \frac{d^{2}}{d t^{2}} q_{i}(t)=-\sum_{i \neq j} \frac{\partial}{\partial q_{i}} \Phi\left(\left|q_{i}(t)-q_{j}(t)\right|\right) \\
& q_{i}(0)=q_{i}, \quad m \dot{q}_{i}(0)=p_{i}, \quad\left\{\left(q_{i}, p_{i}\right)\right\}=x \in \mathfrak{X} .
\end{aligned}
$$

The corresponding formal integral equations of motion are

$$
\begin{aligned}
& q_{i}(t)=q_{i}+p_{i} / m t-\int_{0}^{t} d t_{1}\left(t-t_{1}\right) \sum_{i \neq j} \frac{\partial}{\partial q_{i}} \Phi\left(\left|q_{i}\left(t_{1}\right)-q_{j}\left(t_{1}\right)\right|\right) \\
& \left\{\left(q_{i}, p_{i}\right)\right\}=x \in \mathfrak{X} .
\end{aligned}
$$

We will prove the existence of dynamics by showing the following

Theorem 2.1. Let $\Phi$ satisfy $D 2.2$ and $\mu$ D2.3. Then there exist $\mathfrak{X}^{0}$ and $S(t)$ such that

(i) $\mathfrak{X}^{0}$ has full $\mu$-measure,

(ii) $S(t): \mathfrak{X}^{0} \mapsto \mathfrak{X}^{0}$ for every real $t$ and it is a representation of the real line onto the invertible mappings of $\mathfrak{X}^{0}$ onto itself,

(iii) $x(t)=S(t) x \equiv\left[q_{i}(t), p_{i}(t)\right]$ satisfy the equations of motion D2.4 with initial condition $x \in \mathfrak{X}^{0}$, 
(iv) for every fixed $x \in \mathfrak{X}^{0}$ and $\tau \in \mathbb{R}^{+}$there exist $A(x, \tau) B(x, \tau)$ s.t.

$$
\begin{gathered}
\sup _{\imath}\left(\left|q_{i}(t)-q_{i}\right|\right) \leqq A(x, \tau)\left[\log _{+}\left|q_{i}\right|\right]^{1 / 2} \quad|t| \leqq \tau, \\
\inf _{\llcorner} \inf _{i \neq j}\left(\left|q_{i}(t)-q_{j}(t)\right|\right) \geqq B(x, \tau)\left[\log _{+}\left|q_{i}\right|\right]^{1 / \lambda_{1}}+a \quad|t| \leqq \tau .
\end{gathered}
$$

Uniqueness holds in the class of solutions of the equations of motion satisfying the conditions 2.2.

(v) $S(t)$ is a measurable transformation preserving $\mu$.

The idea of the proof is to introduce partial flows $S^{\alpha}(t)$ under which only a finite number of particles move. Then to take the limit for $\alpha$ going to infinity, so that more and more particles are allowed to move. The existence of the limit is the crucial step in this procedure. Once we prove this it is quite easy to show that the limiting flow $S(t)$ has the desired properties, in a completely similar way to $[2-5]$.

D2.5 Partial Flows. $\sum_{\alpha}$ is the sphere centered in the origin of $\mathbb{R}^{v}$ and with radius $2^{\alpha}, \alpha \in \mathbb{Z}^{+} . S^{\alpha}$ is the partial flow $[2,10]$ in which the particles outside $\sum_{\alpha}$ are frozen, those inside move pairwise interacting in the field of the external ones and when they reach the boundaries of $\sum_{\alpha}$ they are elastically reflected. Problems could arise due to the impulsive forces arising from the collisions. We will need the following lemma $[2,10]$.

Lemma 2.1. For every fixed $y \in \mathfrak{X}\left(\sum_{\alpha}^{c}\right)$ there is a set $\mathscr{M}^{n}(y)$ in $\sum_{\alpha}^{n} \times \mathbb{R}^{n v}$, for every n, of "full" $d(q)_{n} d(p)_{n}$ measure in which $S^{\alpha}(t)$ is defined with the following properties:

(i) $S^{\alpha}(t)$ is an invertible mapping of $\mathscr{M}^{n}(y)$ onto itself which satisfies the equations of motion with elastic reflections on $\partial \sum_{\alpha}$. The number of collisions is finite for every bounded interval of time.

(ii) $S^{\alpha}(t)$ is imbedded into an invertible map [still denoted by $\left.S^{\alpha}(t)\right]$ of $\tilde{\mathfrak{X}}$ onto itself, the particles outside $\sum_{\alpha}$ are fixed under $S^{\alpha}(t) . S^{\alpha}(t)$ is defined in a set of full $\mu$ measure for all the values of $\alpha$ and $t$ and $\mu$ is $S^{\alpha}(t)$ invariant.

\section{Statistical Estimates}

In this section we establish estimates on the displacements of the particles and on maximum closeness between them under the partial flows $S^{\alpha}$.

We do not obtain uniformity in $\alpha$ at this stage, but it will be the same existence of the limiting flow $S(t)$ which will ensure it, as it will be seen in the next section.

The main feature of the estimates we obtain lies in their statistical nature. We could not obtain a proposition stating that if initially the particles are not too close and their velocities not too large, the same will hold for later times. We simply prove that the probability for this to happen is very small and with probability one we can pick up configurations with good behaviour w.r.t. $\alpha$ definitely in $\alpha$. 
Theorem 3.1. Let $\Phi$ satisfy $D 2.2$ and $\mu$ D2.3. Then there exists $c_{1}$ such that the sets

$$
F\left(c_{1}, \tau\right)=\left\{x \in \mathfrak{X}\left|\exists \beta(x)>0: \forall \alpha \geqq \beta(x) \sup _{|t| \leqq \tau} \sup _{q_{i} \in \sum_{\alpha} \cap \hat{q}_{x}}\right| p_{i}^{\alpha}(t) \mid \leqq c_{1}(\alpha)^{1 / 2} ; \tau>0\right\}
$$

have full $\mu$ measure, $\mu\left[F\left(c_{1}, \tau\right)\right]=1$.

Theorem 3.2. Let $\Phi$ and $\mu$ as in Theorem 3.1. Then there exists $c_{2}>0$ such that the sets

$$
\begin{aligned}
G\left(c_{2}, \tau\right)=\{x \in \mathfrak{X} \mid \exists \gamma(x)>0: \forall \alpha & \geqq \gamma(x) \inf _{|t| \leqq \tau} \inf _{q, q^{\prime} \in \sum_{\alpha} \cap \hat{q}_{x}}\left|q^{\alpha}(t)-q^{\prime \alpha}(t)\right| \\
& \left.\geqq a+c_{2} \alpha^{-1 / \lambda_{1}} ; \tau>0\right\}
\end{aligned}
$$

have full $\mu$ measure, $\mu\left[G\left(c_{2}, \tau\right)\right]=1$.

Corollary 3.1. In the hypotheses of Theorem 3.1 the set

$$
\mathscr{I}^{0}=\bigcap_{\tau \geqq 0} F\left(c_{1}, \tau\right) \cap G\left(c_{2}, \tau\right)
$$

has full equilibrium measure, $\mu\left(\mathscr{I}^{0}\right)=1$.

Proof. Since $\left\{F\left(c_{1}, \tau\right)\right\}$ and $\left\{G\left(c_{2}, \tau\right)\right\}$ are decreasing families of sets as $\tau$ increases the corollary is just a consequence of Theorems 3.1 and 3.2 together with countable additivity of $\mu$.

In the next section we will prove that for all the configurations in $\mathscr{I}^{0}$ the equations of motion have solution, and starting from $\mathscr{I}^{0}$ we will construct the set $\mathfrak{X}^{0}$ of Theorem 2.1 .

\section{Proof of the Existence Theorem}

We first prove that $\lim _{\alpha \rightarrow \infty} S^{\alpha}(t) x$ exists (particle by particle) if $x \in \mathscr{I}^{0}$ [Corollary 3.1] and this defines a flow $S(t)$. Then it is easy to show that $S(t) x$ satisfies the equations of motion and the proof of Theorem 2.1 can be completed.

\section{Existence of the Limit of the Partial Flows}

We fix $\tau \in \mathbb{R}^{+}$and $x \in \mathscr{I}^{0}$. Let $\Delta \subset \mathbb{R}^{v}$

$$
\Delta=\sum(0, \delta)=\text { sphere in } \mathbb{R}^{v} \text { centered in } 0 \text { with radius } \delta .
$$

We assume

$$
\delta \geqq \max \left\{2^{\beta(x)}, 2^{\gamma(x)}\right\}
$$

where $\beta(x)$ and $\gamma(x)$ are defined in Theorems 3.1 and 3.2 respectively. We will prove that

$$
\begin{aligned}
& \sum_{2}^{\infty}\left\|q^{\alpha}(t)-q^{\alpha-1}(t)\right\|_{\Delta}<\infty, \\
& \left\|q^{\beta}(t)\right\|_{\Sigma}=\sup _{q_{1} \in \hat{q}_{x} \cap \Sigma}\left|q_{i}^{\beta}(t)\right| \quad \sum \subset \mathbb{R}^{v} .
\end{aligned}
$$


Since $\delta$ can be chosen arbitrarily large, once we prove Eq. (4.3) we obtain

$$
\lim _{\alpha \rightarrow \infty} q_{i}^{\alpha}(t) \equiv q_{i}(t) .
$$

By use of Lemma 2.1 we obtain from the integral equations for $q_{i}^{\alpha}(t)$ and $q_{i}^{\alpha-1}(t)$; $q_{i} \in \Delta,|t| \leqq \tau$

where

$$
\begin{aligned}
\left|q_{i}^{\alpha}(t)-q_{i}^{\alpha-1}(t)\right| \leqq & \int_{0}^{t} d t_{1}\left(t-t_{1}\right)\left\{\sum_{j \in \Delta_{1}(i)} \mid F\left[q_{i}^{\alpha}\left(t_{1}\right)-q_{j}^{\alpha}\left(t_{1}\right)\right]-F\left[q_{i}^{\alpha-1}\left(t_{1}\right)-q_{j}^{\alpha-1}\left(t_{1}\right)\right]\right. \\
& \left.+\sum_{j \notin \Delta_{1}(i)}\left|F\left[q_{i}^{\alpha}\left(t_{1}\right)-q_{j}^{\alpha}\left(t_{1}\right)\right]-F\left[q_{i}^{\alpha-1}\left(t_{1}\right)-q_{j}^{\alpha-1}\left(t_{1}\right)\right]\right|\right\}
\end{aligned}
$$

$$
\begin{aligned}
& F(x)=-\frac{\partial}{\partial x} \Phi(|x|) \quad x \in \mathbb{R}^{v}, \\
& \Delta_{1}(i)=\left\{j \in \mathbb{Z} \mid q_{j} \in \hat{q}_{x} \cap \sum\left[q_{i} ; 2 c_{1}(\alpha)^{1 / 2} \tau+R(\alpha)\right]\right\}
\end{aligned}
$$

[see Eq. (4.1) for def. of $\sum$ and Theorem 3.1 for $c_{1}$ ]

$R(\alpha)$ will be determined later [Eq. (4.15a)] .

In Eq. (4.5) we assumed that the particle " $i$ " does not reach the boundaries of $\sum_{\alpha-1}$ therefore, see Theorem 3.1, we require that

$$
2^{\alpha-1}>\delta+c_{1}(\alpha)^{1 / 2} \tau \text {. }
$$

The second sum in the r.h.s. of Eq. (4.5) is small if $R(\alpha)$ is sufficiently large, this because

$$
\text { for } \beta=\alpha, \alpha-1 \quad\left|q_{j}^{\beta}(t)-q_{i}^{\beta}(t)\right| \geqq R(\alpha) \quad \text { for } \quad|t| \leqq \tau ; j \notin \Delta_{1}(i)
$$

where Theorem 3.1 has been used. Assuming that

$$
R(\alpha)>r_{2}
$$

and taking into account the hard core condition, explicit bounds on the force can be obtained, see D2.2; there exists $B$ sufficiently large so that

$$
\sum_{j \notin \Delta_{1}(i)}\left|F\left[q_{i}^{\alpha}\left(t_{1}\right)-q_{j}^{\alpha}\left(t_{1}\right)\right]\right| \leqq 1 / 2 B[R(\alpha)]^{-\lambda^{\prime}-1+v} \text {, the same for } \alpha-1
$$

where $v$ is the number of dimensions of the space in which the particles move. The details are given in Lemma A 1 in the Appendix.

To estimate the first sum in the r.h.s. of Eq. (4.5) we can use the Lipschitz condition on the pair force, D 2.2. By Theorem 3.2, in fact, the particles remain at distance larger than $c_{2} \alpha^{-1 / \lambda_{1}}$, and this determines the value of the Lipschitz constant effective in our case. As a matter of fact only a few particles, due to the hard-core condition, can simultaneously be so close to the particle $q_{i}$. Taking this into account we prove in Lemma A2 that there exists $A$ sufficiently large so that

$$
\begin{aligned}
& \sum_{j \in \Delta_{1}(i)}\left|F\left[q_{i}^{\alpha}\left(t_{1}\right)-q_{j}^{\alpha}\left(t_{1}\right)\right]-F\left[q_{i}^{\alpha-1}\left(t_{1}\right)-q_{j}^{\alpha-1}\left(t_{1}\right)\right]\right| \leqq A \alpha^{2 u}\left\|q^{\alpha}\left(t_{1}\right)-q^{\alpha-1}\left(t_{1}\right)\right\|_{\Delta_{1}}, \\
& 2 u=\lambda_{1}^{-1}\left(2+\lambda_{2}\right), \\
& \Delta_{1}=\sum\left(0, \delta_{1}\right) \quad \delta_{1}=\delta+\left(2 c_{1}(\alpha)^{1 / 2} \tau+R(\alpha)\right)
\end{aligned}
$$


Equations (4.9) and (4.10) allow to obtain from Eq. (4.5)

$$
\left\|q^{\alpha}(t)-q^{\alpha-1}(t)\right\|_{\Delta} \leqq B[R(\alpha)]^{-\lambda^{\prime}}(2 !)^{-1} \tau^{2}+\int_{0}^{t} d t_{1}\left(t-t_{1}\right) A \alpha^{2 u}\left\|q^{\alpha}\left(t_{1}\right)-q^{\alpha-1}\left(t_{1}\right)\right\|_{\Delta_{1}} .
$$

we can terate the procedure leading to Eq. (4.11) in the hypothesis that the analogous of Eq. (4.7) still holds. To iterate $n$ times we need that

$$
2^{\alpha-1}>\delta_{n}=\delta+n\left[2 c_{1}(\alpha)^{1 / 2} \tau+R(\alpha)\right]
$$

which is the analogous of Eq. (4.7) taking into account Eq. (4.10b).

For the values of $n$ satisfying Eq. (4.12), the $n$-iterated of Eq. (4.11) gives

$$
\begin{aligned}
& \left\|q^{\alpha}(t)-q^{\alpha-1}(t)\right\|_{\Delta} \leqq B R(\alpha)^{-\lambda^{\prime}} \sum_{l=1}^{n}(2 l !)^{-1} \tau^{2 l}\left[A \alpha^{2 u}\right]^{l-1}+\left[A \alpha^{2 u}\right]^{n} \\
& \cdot \int_{0}^{t} d t_{1}\left(t-t_{1}\right) \ldots \int_{0}^{t_{n}-1} d t_{n}\left(t_{n-1}-t_{n}\right)\left\|q^{\alpha}\left(t_{n}\right)-q^{\alpha-1}\left(t_{n}\right)\right\|_{\Delta_{n}} \\
& \quad=B R(\alpha)^{-\lambda^{\prime}} \sum_{l=1}^{n}(2 l !)^{-1} \tau^{2 l}\left[A \alpha^{2 u}\right]^{l-1}+(2 n !)^{-1} \tau^{2 n}\left[A \alpha^{2 u}\right]^{n} 2 c_{1}(\alpha)^{1 / 2} \tau
\end{aligned}
$$

where Theorem 3.1 has been used to estimate $\left\|q^{\alpha}\left(t_{1}\right)-q^{\alpha-1}\left(t_{1}\right)\right\|_{\Delta_{n}}$ :

$$
\begin{aligned}
\left\|q^{\alpha}\left(t_{1}\right)-q^{\alpha-1}\left(t_{1}\right)\right\|_{\Delta_{n}} & \leqq\left\|q^{\alpha}\left(t_{1}\right)-q\right\|_{\Delta_{n}}+\left\|q^{\alpha-1}\left(t_{1}\right)-q\right\|_{\Delta_{n}} \\
& \leqq c_{1}(\alpha)^{1 / 2} \tau+c_{1}(\alpha-1)^{1 / 2} \tau \leqq 2 c_{1}(\alpha)^{1 / 2} \tau .
\end{aligned}
$$

We will show that an opportune choice of $R(\alpha)$ and $n$ in Eq. (4.13), within the restriction of Eq. (4.12), makes the r.h.s. of Eq. (4.13) small as $\alpha \rightarrow \infty$. We have

$$
\begin{aligned}
& B R(\alpha)^{-\lambda^{\prime}} \sum_{l=1}^{n}(2 l !)^{-1} \tau^{2 l}\left[A \alpha^{2 u}\right]^{l-1} \\
& \leqq B R(\alpha)^{-\lambda^{\prime}}\left[A \alpha^{2 u}\right]^{-1} \sum_{l=1}^{\infty}(2 l !)^{-1} \tau^{2 l}\left[A \alpha^{2 u}\right]^{l} \\
& \leqq B R(\alpha)^{-\lambda^{\prime}}\left[A \alpha^{2 u}\right]^{-1} \sum_{l=0}^{\infty}(l !)^{-1}\left[\tau(A)^{1 / 2} \alpha^{u}\right]^{l} \\
& =B R(\alpha)^{-\lambda^{\prime}}\left[A \alpha^{2 u}\right]^{-1} \exp \left[\tau(A)^{1 / 2} \alpha^{u}\right] .
\end{aligned}
$$

We therefore choose

$$
R(\alpha)=2^{\alpha / 3} .
$$

Accordingly we set $n$ in Eq. (4.13) equal to

$$
n(\alpha)=2^{\alpha / 3}
$$

so that Eq. (4.12) is satisfied for $\alpha$ larger than some fixed value. Writing Eq. (4.13) with the estimate Eq. (4.14) and using Eq. (4.15)

$$
\begin{aligned}
\left\|q^{\alpha}(t)-q^{\alpha-1}(t)\right\|_{\Delta} \leqq & \left(B A^{-1}\right) \alpha^{-2 u} 2^{-\alpha \lambda^{\prime} / 3} \exp \left[\tau(A)^{1 / 2} \alpha^{u}\right] \\
& +\left(2 c_{1} \tau\right)(\alpha)^{1 / 2}\left[A \tau^{2}\right]^{n(\alpha)}[2 n(\alpha) !]^{-1} \alpha^{2 u n(\alpha)}
\end{aligned}
$$

which goes to zero as $\alpha$ diverges because $u<1$, see D2.2. 


\section{Bounds on Displacements}

We fix as before $x \in \mathscr{I}^{0}$ and $\tau$. We want to prove that there exists $K$, depending on $x$ and $\tau$, such that for all " $i$ "

$$
\left|q_{i}^{\alpha}(t)-q_{i}\right| \leqq K \alpha_{i} \quad 2^{\alpha_{i}-1} \leqq\left|q_{i}\right| \leqq 2^{\alpha_{2}} .
$$

We choose an integer $v$ depending on $x$ and $\tau$ such that the following holds

(i) $v>\beta(x), \gamma(x)$ [see Eq. (4.2)],

(ii) the sum [over $\alpha$ ] of the r.h.s. of Eq. (4.16) for $\alpha \geqq v$ is less than 1,

(iii) Equation (4.12) holds with $\delta=2^{\alpha-2}$ for $\alpha \geqq v$.

For "small" $\alpha$ Eq. (4.17) is derived directly from Theorem 3.1:

$$
\left|q_{i}^{\alpha}(t)-q_{i}\right| \leqq c_{1}(\alpha)^{1 / 2} \tau \leqq\left(c_{1} \tau v\right)\left(\alpha_{i}\right)^{1 / 2} \alpha \leqq \beta_{i}=\max \left[v, \alpha_{i}\right] .
$$

For large $\alpha, \alpha>\beta_{i}$, we use Eq. (4.16) so that, by the choice of $v$, we have with $\delta=2^{\alpha_{i}}$

$$
\begin{aligned}
\left|q_{i}^{\alpha}(t)-q_{i}\right| & \leqq\left\|q^{\alpha}(t)-q\right\|_{\Delta} \\
& \leqq\left\|q^{\beta_{i}+1}(t)-q\right\|_{\Delta}+\left\|q^{\beta_{2}+2}(t)-q^{\beta_{i}+1}(t)\right\|_{\Delta}+\ldots+\left\|q^{\alpha}(t)-q^{\alpha-1}(t)\right\| \\
& \leqq c_{1} \tau\left(\beta_{i}+1\right)^{1 / 2}+1 \leqq c_{1} \tau(v+1)\left(\alpha_{i}+1\right)^{1 / 2}
\end{aligned}
$$

Equations (4.18) and (4.19) give Eq. (4.17).

\section{Bounds on Closeness between Particles}

The proof is similar to the previous one. We choose $v$ so that

(i)' $v \geqq v$,

(ii)' the sum of the r.h.s. of Eq. (4.16) for $\alpha \geqq v$ is less than $1 / 4 c_{2}(\alpha-1)^{-1 / \lambda_{1}}$,

(iii) $2^{\alpha+1}-2^{\alpha} \geqq 2 A(x, \tau)(\alpha+2)^{1 / 2}+c_{2} \alpha^{-1 / \lambda_{1}}$ for $\alpha \geqq v$.

Condition (ii)' is the analogous of (ii) while (iii)' ensures that if $\left|q_{j}-q_{i}\right|>2^{\alpha_{i}}$ then the particles cannot be closer than $c_{2} \alpha_{i}^{-1 / \lambda_{1}}$, see Eq. (2.2a). Note that by (i) $v$ satisfies also (iii).

Using Theorem 3.2

$$
\begin{aligned}
\left|q_{i}^{\alpha}(t)-q_{j}^{\alpha}(t)\right| & \geqq \min \left\{c_{2} \alpha^{-1 / \lambda_{1}}, c_{2}\left(\alpha_{i}+1\right)^{-1 / \lambda_{1}}\right\},|t| \leqq \tau \text { and } \alpha \leqq \bar{\beta}_{i} \\
& \geqq c_{2} v^{-1 / \lambda_{1}}\left(\alpha_{i}+1\right)^{-1 / \lambda_{1}}
\end{aligned}
$$

where

$$
\bar{\beta}_{i}=\max \left[\alpha_{i}+1, v\right] .
$$

For $\alpha \geqq \bar{\beta}_{i}+1$ and $\left|q_{j}\right| \leqq 2^{\alpha_{i}+1}$ we have, $\bar{\delta}=2^{\alpha_{i}+1}, \bar{\Delta}=\sum(0, \bar{\delta})$

$$
\begin{aligned}
\left|q_{i}^{\alpha}(t)-q_{j}^{\alpha}(t)\right| \geqq & \min _{\left|q_{j}\right| \geqq \delta}\left\{\left|q_{i}^{\bar{\beta}_{i}+1}(t)-q_{j}^{\bar{\beta}_{i}+1}(t)\right|\right\}-2\left[\left\|q^{\bar{\beta}_{i}+2}(t)-q^{\bar{\beta}_{i}+1}(t)\right\|_{\bar{\Delta}}\right. \\
& \left.+\ldots+\left\|q^{\alpha}(t)-q^{\alpha-1}(t)\right\|_{\bar{u}}\right] \\
\geqq & c_{2}\left(\bar{\beta}_{i}+1\right)^{-1 / \lambda_{1}}-21 / 4 c_{2}\left(\bar{\beta}_{i}+1\right)^{-1 / \lambda_{1}} \\
\geqq & c_{2} / 2(v+1)^{-1 / \lambda_{1}}\left(\alpha_{i}+2\right)^{-1 / \lambda_{1}} .
\end{aligned}
$$

For $\left|q_{j}\right|>2^{\alpha_{i}+1}$ we can use directly (iii)' so that the desired bound, Eq. (2.2b), is obtained. 
$S(t) x$ is a Solution of the Equations of Motion

Let $x \in \mathscr{I}^{0}$. Fix $q_{i} \in \hat{q}_{x}$ and $t$. We have for sufficiently large $\alpha$

$$
q_{i}^{\alpha}(t)=q_{i}+p_{i} t / m+\sum_{j \neq i} \int_{0}^{t} d t_{1}\left(t-t_{1}\right) F\left[q_{i}^{\alpha}\left(t_{1}\right)-q_{j}^{\alpha}\left(t_{1}\right)\right] .
$$

We take the limit of both sides of Eq. (4.20) as $\alpha$ goes to infinity.

The 1.h.s. gives $q_{i}(t)$ as proven before. The main point is that the sum in the r.h.s. is uniformly convergent because of the uniform bounds on the displacements proven before. The thesis is then obtained because also the convergence of $q_{j}^{\alpha}\left(t_{1}\right)$ is uniform in $\left|t_{1}\right| \leqq t$.

The same equations of motion prove that

$$
S\left(t_{1}+t_{2}\right) x=S\left(t_{1}\right) S\left(t_{2}\right) x \quad x \in \mathscr{I}^{0}
$$

which naturally extends $S(t)$ to the set

$$
\mathfrak{X}^{0}=\bigcup_{t \in \mathbb{R}} S(t) \mathscr{I}^{0} \text {. }
$$

$\mathfrak{X}^{0}$ is invariant under $S(t)$ and so also (ii) of Theorem 2.1 is proven. Uniqueness in the sense of (iii) is easily proven with the same iterative procedure used to prove the existence of the limit $S^{\alpha}(t)$, see also $[3,7]$. We still have to prove $(v)$ of Theorem 2.1. The proof is based on the invariance of $\mu$ under the partial flows $S^{\alpha}(t)$ and the existence of the limit for $S^{\alpha}(t)$. Since the analogous proof in $[3,5]$ was based only on these facts we omit it.

\section{Appendix}

In this appendix we first prove Theorems 3.1 and 3.2. The essential point in both theorems is the use of a technique introduced by Sinai [5], to obtain bounds on the velocities of the particles. Since Theorem 3.1 is more similar to the analogous ones in $[2,3,5]$ we firstly present in some details the proof of Theorem 3.2. We then just sketch the main differences one meets in dealing with Theorem 3.1.

\section{Proof of Theorem 3.2}

Let

$$
G^{\alpha}\left(c_{2}, \tau\right)=\left\{x \in \mathfrak{X}\left|\inf _{|t| \leqq \tau} \inf _{\left\{q, q^{\prime} \in \hat{q}_{x} \cap \sum \alpha\right\}}\right| q^{\alpha}(t)-q^{\prime \alpha}(t) \mid \geqq a+c_{2} \alpha^{-1 / \lambda_{1}}\right\} .
$$

We estimate the measure of

$$
B^{\alpha}\left(c_{2}, \tau\right)=\text { complement of } G^{\alpha}\left(c_{2}, \tau\right)
$$

as the sum of $\mu\left[B_{1}^{\alpha}\left(c_{2}, \tau\right)\right]$ and $\mu\left[B_{2}^{\alpha}\left(c_{2}, \tau\right)\right] . B_{1}^{\alpha}$ is the subset of $B^{\alpha}$ in which condition 5.1 is violated at $t=0$, therefore $\mu\left(B_{1}^{\alpha}\right)$ can be estimated just by static considerations. Since the configurations of $B_{1}$ have large energies (for large $\alpha$ ) because of the Gibbs factor, $\mu\left(B_{1}^{\alpha}\right)$ will approach zero as $\alpha$ diverges.

$B_{2}^{\alpha}$ is the set of configurations for which condition 5.1 is not violated at $t=0$ but it will eventually within time $\tau . B_{2}^{\alpha}$ is therefore represented as the evolution of the set $\Gamma$ which has a pair of particles at distance $\left(a+c_{2} \alpha^{-1 / \lambda_{1}}\right)$. Since the 
measure $\mu$ is invariant under $S^{\alpha}$ again the estimate is essentially static: technically we use the Ambrose Kakutani representation of flows [6] with base $\Gamma$ as firstly developed by Sinai [5] in this context. More precisely we have [where $z$ below is a constant representing the fugacity and arising from the chemical potential and the integration on the momenta]

$$
\begin{aligned}
\mu\left[B_{1}^{\alpha}\right] \equiv & \int_{\mathfrak{X}\left[\sum_{\alpha}^{c}\right]} d \mu(y) \sum_{n}(n !)^{-1} z^{n} \int_{\sum_{\alpha}^{n}} d(q)_{n} \exp \left\{-\beta \alpha\left[(q)_{n} \mid y\right]\right\} \chi\left[B_{1}^{\alpha} ;(q)_{n} y\right] \\
\leqq & \int_{\mathfrak{X}\left[\sum_{\alpha}^{c}\right]} d \mu(y) \sum_{n}(n !)^{-1} z^{n} n(n-1) / 2 \int_{\left[\Sigma_{\alpha}\right]^{n-2}} d(q)_{n}^{1,2} \\
& \left.\cdot \exp \left\{-\beta U\left[(q)_{n}^{1,2} \mid y\right)\right]\right\} \int_{\sum_{\alpha}} d q_{1} \int_{\sum_{\alpha}} d q_{2} \exp \left\{-\beta\left(\alpha^{-1 / \lambda_{1}} c_{2}\right)^{-\lambda_{1}}\right\} \\
& \cdot \exp \left\{-\beta\left[I\left(q_{1} \mid(q)_{n}^{1,2} y\right)+I\left(q_{2} \mid(q)_{n}^{1,2} y\right)\right]\right\} \chi\left[\left|q_{2}-q_{1}\right| \leqq c_{2} \alpha^{-1 / \lambda_{1}}+a ; q_{2}\right] \\
\leqq & \int_{\mathfrak{X}\left[\sum_{\alpha}^{c}\right]} d \mu(y) z^{2} / 2 \exp \left[-\beta c_{2}^{-\lambda_{1}} \alpha\right] \exp (2 \beta\|\Phi\|)\left|\sum_{\alpha}\right|^{2} \sum_{n}(n !)^{-1} z^{n} \\
& \cdot \int_{\sum_{\alpha}^{n}} d(q)_{n} \exp \left\{-\beta U\left[(q)_{n} \mid y\right]\right\} \\
\leqq & \text { const. } \exp \left[-\beta c_{2}^{-\lambda_{1}} \alpha+2 \alpha v\right]
\end{aligned}
$$

[see D 2.3 for notations] ${ }^{1}$, where in Eq. (5.3)

$$
(q)_{n}^{i, j, \ldots}=\left(\ldots q_{l} \ldots\right) \quad \text { where } \quad 1 \leqq l \leqq n \quad \text { and } \quad l \neq i, j, \ldots
$$

$\|\Phi\|$ is defined by the following property, see D 2.2 hypotheses,

$$
\text { for every } x \in \mathfrak{X}: \inf _{q \in \hat{q}_{x}} \sum_{q^{\prime} \in \hat{q}_{x}} \Phi\left(\left|q-q^{\prime}\right|\right) \geqq-\|\Phi\| \text {. }
$$

To evaluate $\mu\left(B_{2}^{\alpha}\right)$ we write

$$
\mu\left(B_{2}^{\alpha}\right)=\int_{\mathfrak{X}\left(\sum_{\alpha}^{c}\right)} d \mu(y) \sum_{n}(n !)^{-1} \cdot \int_{\sum_{\alpha}^{n} \times \mathbb{R}^{v n}} d(q)_{n} d(p)_{n} \exp \left\{-\beta H\left[(q)_{n}(p)_{n} \mid y\right]\right\} \chi_{B_{2}^{\alpha}} .
$$

Therefore for any given $y$ outside $\sum_{\alpha}$ we define the flow $S^{\alpha}$ in $\sum_{\alpha}$ in a set of full $d(q)_{n} d(p)_{n}$ measure [see Lemma 2.1] and in this set we consider the configurations which violate Eq. (5.1) only at times different from zero. We therefore introduce for every $n$, number of particles in $\sum_{\alpha}$, a surface $\Gamma^{n}$ in the $n$-particle phase space $\sum_{\alpha}^{n} \times \mathbb{R}^{v n}$ made up by $n(n-1) / 2$ subregions such that in each of them, $\Gamma_{i j}^{n}$, the pair of particles $q_{i}$ and $q_{j}$ are at distance $a+c_{2} \alpha^{-1 / \lambda_{1}}$. The measure $d(q)_{n} d(p)_{n}$ can be projected on $\Gamma^{n}$ along the $S^{\alpha}$ flow to have

$$
d(q)_{n} d(p)_{n}=d \Gamma^{n} d t \quad \text { on } \bigcup_{|t| \leqq \tau} S^{x}(t) \Gamma^{n} .
$$

To write down explicitely $d \Gamma^{n}$ we consider $\mathscr{M}^{n}(y)$ as a differentiable manifold with a local system of coordinates: if $x \in \mathscr{M}^{n}$

$$
x=\left(q_{1}, \ldots, q_{n} ; p_{1}, \ldots, p_{n}\right) .
$$

A metric is introduced as

$$
d q_{1}^{2}+\ldots+d q_{n}^{2}+d p_{1}^{2}+\ldots+d p_{n}^{2} .
$$

The idea is to project $d(q)_{n} d(p)_{n}$ along the normal $\zeta$ to $\Gamma^{n}$ and then obtain $d \Gamma^{n}$ by this and the scalar product between the normal to $\Gamma^{n}$ and the tangent vector (derivative) of the flow, [5, Lemma]. In our case is however simpler to first project $d(q)_{n} d(p)_{n}$ along another direction $w$. More precisely

$$
\begin{gathered}
d(q)_{n} d(p)_{n}=d(q)_{n}^{j} d(p)_{n} d \sigma_{i j} d r_{i j}=d \Gamma_{i j}^{w} d r_{i j}, \\
d(q)_{n} d(p)_{n}=d \Gamma_{i j}^{\zeta} d r_{i j}(w, \zeta)
\end{gathered}
$$

\footnotetext{
Hereafter we will denote by $\chi(B ; x)$ the value in $x$ of the characteristic function of the set $B$.
} 
where $d \sigma_{i j}$ is the Lebesgue measure on $\mathbb{R}^{v}$ projected on a sphere of radius equal to $a+c_{2} \alpha^{-1 / \lambda_{1}}$ and $r_{i j}$ is the distance between $q_{i}$ and $q_{j}$. Of course $w$ is not necessarily normal to $\Gamma_{i j}^{n}$, it is just orthogonal to those particular curves in $\Gamma_{i j}^{n}$ for which $q_{i}$ is constant. However it is easy to see that if $\vartheta$ is a unit vector tangent to $\Gamma_{i j}^{n}$ then

$$
|(\vartheta, w)| \leqq(2)^{-1 / 2}
$$

so that, with $\zeta$ unit normal vector to $\Gamma_{i j}^{n}$

$$
|(\zeta, w)| \geqq(2)^{-1 / 2}
$$

and this gives, together to Eqs. (5.8b) and (5.8a)

$$
d \Gamma^{\zeta}=\text { normal projection of } d(q)_{n} d(p)_{n} \leqq(2)^{1 / 2} d \Gamma^{w} .
$$

To obtain $d \Gamma^{n}$ we consider the scalar product between $\zeta$ and $\pi$, where

$$
\begin{aligned}
\zeta & =\left(0, \ldots, \zeta_{i}, 0, \ldots, \zeta_{j}, 0, \ldots ; 0, \ldots, 0\right), \\
\pi & =\left(\dot{q}_{1}, \ldots, \dot{q}_{n} ; \dot{p}_{1}, \ldots, \dot{p}_{n}\right)=\left(p_{1} / m, \ldots, p_{n} / m ; \dot{p}_{1}, \ldots, \dot{p}_{n}\right) .
\end{aligned}
$$

We then have

$$
|(\pi, \zeta)| \leqq 1 / m\left(\left|p_{i}\right|+\left|p_{j}\right|\right) .
$$

Therefore with the notations of Eq. (5.7) we have, by use of Eqs. (5.8), (5.9), (5.10):

$$
d \Gamma_{i j}^{n} \leqq(2)^{1 / 2} 1 / m\left(\left|p_{i}\right|+\left|p_{j}\right|\right) d(q)_{n}^{j} d(p)_{n} d \sigma_{i j} .
$$

By Eqs. (5.7) and (5.11) we have from Eq. (5.6)

$$
\begin{aligned}
\mu\left(B_{2}^{\alpha}\right) \leqq & \int_{\mathfrak{x}\left(\Sigma_{\alpha}^{\alpha} \alpha\right.} d \mu(y) \sum_{n}(n !)^{-1} \int_{\Gamma^{n}} d \Gamma^{n} \int_{-\tau}^{\tau} d t \exp \left\{-\beta H\left[(q)_{n}(p)_{n} \mid y\right]\right\} \\
\leqq & \int_{\mathfrak{X}\left(\Sigma_{\alpha}^{\varepsilon}\right)} d \mu(y) \sum_{n}(n !)^{-1} n(n-1) / 2 \int_{\Sigma_{\alpha}^{n-2}} d(q)_{n}^{1,2} \int_{\mathbb{R}^{v(n-2)}} d(p)_{n}^{1,2} \\
& \cdot 2 \tau \exp \left\{-\beta H\left[(q)_{n}^{1,2}(p)_{n}^{1,2}|y|\right\} \exp \left\{-\beta\left(c_{2} \alpha^{-1 / \lambda_{1}}\right)^{-\lambda_{1}}\right\}\right. \\
& \cdot \int_{\Sigma_{\alpha}} d q_{1} \int d \sigma_{12} \chi\left[\left|q_{2}-q_{1}\right|=a+c_{2} \alpha^{-1 / \lambda_{1}} ; q_{2}\right] \\
& \cdot \exp \left\{-\beta\left[I\left(q_{1} \mid(q)_{n}^{1,2} y\right)+I\left(q_{2} \mid(q)_{n}^{1,2} y\right)\right]\right\} \\
& \cdot \int_{\mathbb{R}^{v 2}} d(p)_{2} \exp \left\{-\beta T\left[(p)_{2}\right]\right\}\left(\left|p_{1}\right|+\left|p_{2}\right|\right) / m \\
< & \text { const. } \exp \left[\alpha \nu-\beta c_{2}^{-\lambda_{1}} \alpha\right]
\end{aligned}
$$

where invariance of the energy has been taken into account and the last inequality was obtained in a similar way to the derivation of Eq. (5.3).

Therefore if $c_{2}$ is sufficiently small by Eqs. (5.3) and (5.12) we have: there exist constants $K$ and $c$ such that

$$
\left[G^{\alpha}\left(c_{2}, \tau\right)\right]>1-K \exp (-\beta c \alpha) .
$$

Theorem 3.2 is therefore proven with the position

$$
G\left(c_{2}, \tau\right)=\bigcup_{1}^{\infty} \bigcap_{\beta}^{\infty} G^{\alpha}\left(c_{2}, \tau\right) .
$$




\section{Proof of Theorem 3.1}

It is convenient in the following proof to consider

$$
|p|=\max _{1 \leqq \delta \leqq \nu}\left|p_{\delta}\right|
$$

where $p_{\delta}$ is the $\delta$ component of the vector $p$. The results we obtain then automatically hold also in the previous metric.

In analogy with the proof of Theorem 3.2 we consider the sets

$$
F^{\alpha}\left(c_{1}, \tau\right)=\left\{x \in \mathfrak{X}\left|\sup _{|t| \leqq \tau} \sup _{q_{\imath} \in \sum_{\alpha} \cap \hat{q}_{x}}\right| p_{i}^{\alpha}(t) \mid \leqq c_{1} \sqrt{\alpha}\right\} \text {. }
$$

To evaluate the complement of $F^{\alpha}\left(c_{1}, \tau\right)$ we introduce

$$
D_{1}^{\alpha}\left(c_{1}, \tau\right)=\left\{x \in \mathfrak{X}\left|\sup _{q_{\imath} \in \sum_{\alpha} \cap \hat{q}_{x}}\right| p_{i} \mid \geqq c_{1}(\alpha)^{1 / 2}\right\} .
$$

We have

$$
\begin{aligned}
\mu\left(D_{1}^{\alpha}\right)= & \int_{\mathfrak{X}\left(\sum_{\alpha}\right)} d \mu(y) \sum_{n}(n !)^{-1} \int_{\sum_{\alpha}^{n}} d(q)_{n} \exp \left\{-\beta U\left[(q)_{n} \mid y\right]\right\} \int_{\mathbb{R}^{\nu n}} d(p)_{n} \\
& \cdot \exp \left\{-\beta T\left[(p)_{n}\right]\right\} \chi\left[D_{1}^{\alpha} ;(p)_{n}\right] \\
\leqq & \int_{\mathfrak{X}\left(\sum_{\alpha}^{\alpha}\right)} d \mu(y) \sum_{n}(n !)^{-1} \int_{\sum_{\alpha}^{n}} d(q)_{n} \exp \left\{-\beta U\left[(q)_{n} \mid y\right]\right\} n v \int_{\mathbb{R}^{\nu n}} d(p)_{n}^{1} \\
& \cdot \exp \left\{-\beta T\left[(p)_{n}^{1}\right]\right\} \int_{\left|p_{1}\right| \geqq c_{1}(\alpha)}{ }_{1 / 2} d(p)_{1} \exp \left\{-\beta T\left[(p)_{1}\right]\right\} \\
\leqq & \text { const. } \exp \left[-\beta c_{1}^{2} \alpha / 2 m+\alpha \nu\right] .
\end{aligned}
$$

To estimate $D_{2}^{\alpha}$

$$
D_{2}^{\alpha}=\left\{x \in \mathfrak{X}\left|x \notin D_{1}^{\alpha}, \sup _{|t| \leqq \tau} \sup _{\left(q_{i} \in \hat{q}_{x} \cap \Sigma_{\alpha}\right)}\right| p_{i}^{\alpha}(t) \mid \geqq c_{1}(\alpha)^{1 / 2}\right\} .
$$

We then have the analogous of Eq. (5.6), we introduce the surface $\Delta^{n}$ in $\mathscr{M}^{n}(y)$ made up by $v n$ subsets $\Delta_{i, \delta}^{n} \delta=1, \ldots, v i=1, \ldots, n$ so that

$$
\Delta_{i, \delta}^{n}=\left\{\mathrm{x} \in \mathfrak{X}|| p_{i, \delta} \mid=c_{1}(\alpha)^{1 / 2}\right\} .
$$

Then we project $d(q)_{n} d(p)_{n}$ along the flow on $\Delta^{n}$

$$
d(q)_{n} d(p)_{n}=d \Delta^{n} d t
$$

We have, see D 2.3;

$$
d \Delta_{i, \delta}^{n}=d(q)_{n} d(p)_{n}^{i} d\left(p_{i}\right)^{\delta} \exp (\beta \bar{\mu})\left(\pi^{*}, \zeta^{*}\right)
$$

where $d\left(p_{i}\right)^{\delta}=\prod_{\gamma \neq \delta}^{1, v} d p_{i \gamma} . \pi^{*}$ is the vector tangent to the flow as defined before Eq. (5.10) and hereafter denoted by $\pi^{*}$ to distinguish it from the number $\pi$. $\zeta^{*}$ is now the unit vector normal to $\Delta_{i, \delta}^{n}$ :

$\zeta^{*}$ is a vector with non-zero component only along $\delta$ direction, " $i$ " particle. Therefore

$$
\left|\left(\pi^{*}, \zeta^{*}\right)\right|<\left|F_{i}\right| \quad F_{i}=\dot{p}_{i}=\text { force on the " } i \text { " particle } .
$$


Using this in Eq. (5.16), we have in analogy to Eq. (5.12)

$$
\begin{aligned}
\mu\left(D_{2}^{\alpha}\right) \leqq & \int_{\mathfrak{x}\left(\sum_{\alpha}^{c}\right)} d \mu(y)(2 \tau) \sum_{n}(n !)^{-1} z^{n} n v \int_{\sum_{\alpha-1}^{n-1}} d(q)_{n}^{1} \exp \left\{-\beta U\left[[q]_{n}^{1} \mid y\right]\right\} \\
& \cdot\left(2 \pi \beta^{-1} m\right)^{1 / 2} \exp \left\{-\beta c_{1}^{2} \alpha / 2 m\right\} \int_{\Sigma_{\alpha}} d(q)_{1} F\left[q_{1} \mid(q)_{n}^{1} q_{y}\right] \\
& \cdot \exp \left\{-\beta I\left[q_{1} \mid(q)_{n}^{1} q_{y}\right]\right\}
\end{aligned}
$$

where

$$
F\left[q_{1} \mid q_{x}\right]=\sum_{q_{1} \in q_{x}} \frac{\partial}{\partial q_{1}} \Phi\left(\left|q_{1}-q_{i}\right|\right) .
$$

Since $\exp \left\{-\beta I\left(q_{1} \mid q_{x}\right)\right\} F\left(q_{1} \mid q_{x}\right)$ is bounded in $q_{1}$ and $x$, there exist a constant $K$ such that

$$
\int_{\Sigma_{\alpha}} d q_{1} \exp \left\{-\beta I\left(q_{1} \mid q_{x}\right)\right\} F\left(q_{1} \mid q_{x}\right)<K\left|\sum_{\alpha}\right|
$$

we have

$$
\begin{aligned}
\mu\left(D_{2}^{\alpha}\right)< & \int_{\mathfrak{F}\left(\sum_{\alpha}^{c}\right)} d \mu(y) \exp \left[-\beta c_{1}^{2} \alpha / 2 m\right]\left(2 \pi \beta^{-1} m\right)^{1 / 2}(2 \tau) z K\left|\sum_{\alpha}\right| \sum_{n} z^{n}(n !)^{-1} \\
& \cdot \int_{\sum_{\alpha}^{n}} d(q)_{n} \exp \left\{-\beta U\left[(q)_{n} \mid y\right]\right\} \\
\leqq & \text { const. } \exp \left[-\beta c_{1}^{2} \alpha / 2 m+\alpha \nu\right]
\end{aligned}
$$

which goes exponentially to zero if $c_{1}$ is sufficiently large. The definition of $F\left(c_{1}, \tau\right)$ is now perfectly analogous to that given for $G\left(c_{2}, \tau\right)$ after Eq. (5.12).

In the remaining of this appendix we will give the proofs of some estimates needed in Section 4.

Lemma 5.1. Let $q_{x}$ be the generic spatial configuration in $\mathfrak{X}$ such that $q_{0}=0$, $q_{i} \in \sum(0, R)^{c}$ for $i \neq 0$ and $r_{2}<R$. Then there exists a constant $B$ independent of $q_{x}$ such that

$$
\sum_{i \neq 0}\left|F\left(q_{0}-q_{i}\right)\right| \leqq B R^{-\lambda^{\prime}-1+v} .
$$

Proof. Since $R>r_{2}$ by use of D 2.2 we have, $\left(q_{0}=0\right)$,

$$
\left|F\left(q_{0}-q_{i}\right)\right| \leqq\left|q_{i}\right|^{-1-\lambda^{\prime}} \text {. }
$$

Because the r.h.s. is decreasing with the distance of the particles

$$
\sum_{i \neq 0}\left|F\left(q_{0}-q_{i}\right)\right| \leqq \sum_{r \in \mathbb{Z}^{v}}|\underline{a} r|^{-1-\lambda^{\prime}}, \quad \text { for } \quad|\underline{a} r| \geqq R-a
$$

where $\underline{a}=a / 10$. Therefore

$$
\sum_{i \neq 0}\left|F\left(q_{0}-q_{i}\right)\right| \leqq(\underline{a})^{-1} \int_{|x|>R-10 a} d x x^{-1-\lambda^{\prime}} \leqq B R^{-\lambda^{\prime}-1+v} .
$$

Lemma 5.2. There exists $A$ such that the following holds. Let $\left\{q_{i}\right\}$ and $\left\{q_{i}^{\prime}\right\}$ be spatial configurations with $q_{0}=q_{0}^{\prime}$ and for $i \neq 0\left|q_{i}\right|>r>a$. Then

$$
\sum_{j}\left|F\left(q_{i}\right)-F\left(q_{i}^{\prime}\right)\right| \leqq A r^{-2-\lambda_{2}}\left\{\sup _{j}\left|q_{j}-q_{j}^{\prime}\right|\right\} \quad q_{0}=q_{0}^{\prime}=0 .
$$


Proof. By D2.2 there exists a continuous non increasing function $k(|x|)$, $x \in \mathbb{R}^{v}$, on $[r, \infty)$ such that

$$
\begin{array}{ll}
\frac{d^{2}}{d x^{2}} \Phi(x) \mid \leqq k(|x|), & \\
k(|x|)=k(|x|-a)^{-2-\lambda_{2}} & |x| \leqq r_{1}, \\
k(|x|)=k|x|^{-\lambda^{\prime}} & |x| \geqq r_{2} .
\end{array}
$$

Let $x$ and $y \in \mathbb{R}^{v}$ then

$$
F(y)-F(x)=\int_{\gamma(x, y)} d l \frac{d}{d l} F[x(l)]
$$

where $d l$ is the arc length along the curve $\gamma$, and $\gamma(x, y)$ is a regular curve connecting $x$ and $y$ such that its distance from the origin is either $|x|$ or $|y|$. Further we may and will require that

$$
|\gamma(x, y)| \leqq 2 \pi|x-y|
$$

Therefore since $k$ is a monotone function

$$
\begin{aligned}
|F(y)-F(x)| & \leqq \int_{\gamma(x, y)} d l k(|x(l)|) \leqq[k(|x|)+k(|y|)]|\gamma(x, y)| \\
& \leqq 2 \pi[k(|x|)+k(|y|)]|x-y| .
\end{aligned}
$$

Then we have

$$
\begin{aligned}
\sum_{i}\left|F\left(q_{i}\right)-F\left(q_{i}^{\prime}\right)\right| & \leqq \sum_{i \neq 0}\left[k\left(\left|q_{i}\right|\right)+k\left(\left|q_{i}^{\prime}\right|\right)\right] 2 \pi\left|q_{i}-q_{i}^{\prime}\right| \\
& \leqq 2 \pi\left[\sup _{i}\left|q_{i}-q_{i}^{\prime}\right|\right] \cdot \sum_{i}\left[k\left(\left|q_{i}\right|\right)+k\left(\left|q_{i}^{\prime}\right|\right)\right] .
\end{aligned}
$$

The sum in the r.h.s. can be split into two parts; the contribution of the particles in the sphere $\sum(0,10 a)$ which is bounded by $N$ [the largest number of particle in the sphere] times the maximum value of $k(|q|)$, namely $k(r)$. The second sum is finite due to the explicit form of $k(\cdot)$ so that

$$
\sum_{i} k\left(\left|q_{i}\right|\right) \leqq N k(r)+\sum_{q_{i} \notin \Sigma} k\left(\left|q_{i}\right|\right) \leqq N r^{-2-\lambda_{2}} .
$$

From this the thesis is easily obtained.

Acknowledgment. We are deeply indebted and grateful to the Referee of the Commun. Math. Phys. for pointing out uncountably many errors in a previous version of this paper, [1]. One of us (E.P.) acknowledges very kind hospitality at the Math. Dept. of the Stanford University.

Note Added in Proof. After this paper was completed we realized that analogous results have been obtained for the one dimensional case by Semliakow in a paper published in "Uspekhi Matematiceski Nauk., XXVIII, Vol. 1, 1973".

\section{References}

1. Presutti,E., Pulvirenti,M., Tirozzi,B.: An existence theorem for the equations of motion of infinitely many one-dimensional hard cores with long range two body interactions. Nota interna n. 544, Istituto Fisico Universita' di Roma 1974 
2. Sinai, Ya.G.: The construction of cluster dynamics for dynamical systems of statistical mechanics. Vest. Moskow Univ. 1, 152 (1974)

3. Marchioro,C., Pellegrinotti,A., Presutti,E.: Time evolution in statistical mechanics. Lettere al N.C. 11, 606 (1974)

Marchioro,C., Pellegrinotti,A., Presutti,E.: Existence of time evolution for $v$ dimensional statistical mechanics. Commun. math. Phys. 40, 175 (1975)

4. Lanford III,O.E.: Dynamics of systems of infinitely many particles: existence. Battelle Conference. Seattle 1974

5. Sinai, Ya.G.: Construction of dynamics in one dimensional systems of infinitely many particles. Sov. Theor. Math. Phys. 12, 487 (1973)

6. Ambrose, W.: Representation of ergodic flows. Ann. Math. 42, 723 (1942)

Ambrose, W., Kakutani, S.: Structure and continuity of measurable flows. Duke Math. J. 9, 25 (1942)

7. Lanford III,O.E.: The classical mechanics of one dimensional systems of infinitely many particles. Commun. math. Phys. 9, 176 (1968)

Lanford III,O.E.: The classical mechanics of one dimensional systems of infinitely many particles: II kinetic theory. Commun. math. Phys. 11, 257 (1969)

8. Ruelle, D.: Statistical mechanics: rigorous results. New York: Benjamin 1969

9. Dobrushin, R. L.: Gibbsian probability fields. Funkts. Anal. Ego Pril. 2, 31 (1968); 2, 44 (1968); 3, 27 (1969)

Lanford III,O.E., Ruelle, D.: Observables at infinity and states with short range correlations in statistical mechanics. Commun. math. Phys. 13, 194 (1969)

Ruelle, D.: Superstable interactions in classical statistical mechanics. Commun. math. Phys. 18, 127 (1970)

10. Marchioro, C., Pellegrinotti, A., Presutti, E., Pulvirenti, M.: On the dynamics of particles moving in bounded regions: a measure theoretical approach. Nota interna, Istituto Matematico Università Roma, 1974

Communicated by J. L. Lebowitz

(Received May 17, 1974)

\section{Erratum}

Gallavotti,G., Pulvirenti, M.: Classical KMS Condition and Tomita-Takesaki Theory. Commun. math. Phys. 46, 1-9 (1976)

Date of receipt: March 9, 1975; in revised form July 23, 1975 
